\title{
Central versus subtotal pancreatectomy: an assessment of short and long-term outcomes
}

\author{
Kunal Hanchate, Mary Tice, Tamanie E. Yeager, John A. Stauffer \\ Department of Surgery, Mayo Clinic, Jacksonville, Florida, USA \\ Contributions: (I) Conception and design: K Hanchate, M Tice, JA Stauffer; (II) Administrative support: M Tice, TE Yeager, JA Stauffer; \\ (III) Provision of study materials or patients: K Hanchate, JA Stauffer; (IV) Collection and assembly of data: K Hanchate, JA Stauffer; (V) Data \\ analysis and interpretation: K Hanchate, M Tice, TE Yeager, JA Stauffer; (VI) Manuscript writing: All authors; (VII) Final approval of manuscript: \\ All authors. \\ Correspondence to: John A. Stauffer, MD. Division of General Surgery, Mayo Clinic, 4500 San Pablo Road, Jacksonville, Florida 32224, USA. \\ Email: stauffer.john@mayo.edu.
}

Background: Operative options for low-grade lesions of the pancreas neck include central pancreatectomy (CP) and subtotal pancreatectomy (SP). We intend to directly compare the outcomes between CP and SP.

Methods: A retrospective review was undertaken to include all pancreas resections performed from October 2010 to April 2019 at our institution. Patients who underwent CP were matched for sex, BMI, age, surgical date, and tumor characteristics to 10 patients undergoing SP. Outcome variables included: postoperative pancreatic fistula (POPF), hemorrhage (PPH), delayed gastric emptying (DGE), the occurrence of long-term endocrine and exocrine insufficiency, and overall morbidity as assessed by the Clavien scale.

Results: Ten patients underwent central resection. Both CP and SP resulted in definitive treatment and there were no mortalities. CP was associated with significantly longer operative time, longer hospital stays, significantly higher rate of POPF and major morbidity. At mean follow up of 79.7 months, a higher proportion of patients undergoing SP developed new onset DM and required PERT but was not statistically significant.

Conclusions: While patients undergoing SP were shown to fare better than those undergoing CP in terms of short-term outcomes, there is no apparent significant advantage with either operation regarding longterm exocrine or endocrine insufficiencies.

Keywords: Pancreatectomy; subtotal pancreatectomy (SP); central pancreatectomy (CP); pancreatic cancer

Received: 03 March 2021; Accepted: 22 June 2021; Published: 20 July 2021.

doi: 10.21037 /jovs-21-16

View this article at: https://dx.doi.org/10.21037/jovs-21-16

\section{Introduction}

Pancreatic lesions often require precise surgical resection that is associated with technical difficulty and major morbidity. Pancreatectomy techniques used for lowgrade lesions localized in pancreas neck include central pancreatectomy (CP) and subtotal pancreatectomy (SP). $\mathrm{CP}$ involves the removal of the proximal body/neck of the pancreas and requires reconstruction of the left side of the pancreas into the GI tract. In comparison, SP is a near total pancreatectomy that involves the neck, body and tail of the pancreas and leaves only the head and uncinate process intact. CP leaves more intact parenchyma and may be associated with less endocrine and exocrine insufficiency than extended resection techniques such as SP. On the contrary, $\mathrm{CP}$ does tend to have a high complication rate with postoperative pancreatic fistula (POPF) arising from the GI reconstruction $(1,2)$.

While there is literature comparing the outcomes and efficacy of CP and distal pancreatectomy, there is none directly comparing CP and SP. We sought to understand the surgical and medical complications of CP and SP. In this study, the short-term complications and long-term 
endocrine and exocrine insufficiencies of SP and $\mathrm{CP}$ are assessed and compared. We present the following article in accordance with the STROBE reporting checklist (available at https://jovs.amegroups.com/article/view/10.21037/jovs$21-16 / \mathrm{rc})$.

\section{Methods}

A retrospective review was undertaken for patients undergoing pancreatic resection from October 2010 to April 2019 in Mayo Clinic, Florida. The study was conducted in accordance with the Declaration of Helsinki (as revised in 2013). The study was approved by institutional review board (IRB\#21-005622) and individual consent for this retrospective analysis was waived.

The patients who underwent $\mathrm{CP}$ were matched by sex, $\mathrm{BMI}$, age, and underlying pathology to 10 patients in whom SP was performed in the same time frame. Variable data was obtained by our comprehensive electronic medical record that included all visits to not only the surgical teams - patients were routinely screened for endocrine and exocrine dysfunction preoperatively and postoperatively. Additionally, review of consultations with gastroenterology, endocrinology, and primary care providers was performed. No missing data was identified.

Indications for pancreatectomy included lesions (cystic and solid) and benign strictures localized at the neck or proximal body of the pancreas. Appropriateness for CP and SP was determined by imaging modalities and diagnostic endoscopic procedures (computed tomography, magnetic resonance imaging, endoscopic ultrasound, and endoscopic retrograde cholangiopancreatography). Decisions for surgical resection were performed in a multidisciplinary fashion.

Patient information such as demographics, operative variables, postoperative outcomes, and long-term outcomes of endocrine and exocrine insufficiency were assessed. Postoperative complications were scored to include those within 90 days of surgery including POPF, postoperative pancreatic hemorrhage (PPH), delayed gastric emptying (DGE), and morbidity as assessed by the Clavien scale. Major morbidity was defined as those patients with grade III or higher complications. POPF (3), PPH (4), and DGE (5) were scored according to the current standard international consensus definitions. Long-term endocrine and exocrine insufficiency are defined as occurrence greater than 6 months post-operation. Endocrine insufficiency is assessed through diabetes mellitus (DM) status pre-op and postop and subsequent diabetic status conversion. Pre-diabetic (diet-controlled) patients were considered diabetic for the purpose of this study. Use of pancreas enzyme replacement therapy (PERT) and incidence of steatorrhea was used to assess exocrine insufficiency.

\section{Statistical analysis}

Continuous variables are reported as mean (SD) and categorical variables are presented as number (percentage). Standard statistical analysis was performed. Significance is noted at $\mathrm{P}<0.05$.

\section{Surgical technique}

All surgeons were fellowship trained in hepatopancreatobiliary surgery at an institution with high-volume of pancreatic surgeries, and in previous studies have demonstrated low POPF rates as a practice $(6,7)$. For SP, all operations were performed using the clockwise technique as described previously (8). Briefly, a four-trocar technique with wide exposure of the pancreas through the lesser sac was used. The superior mesenteric vein was identified, and a tunnel was made under the neck of the pancreas. The common hepatic artery was dissected away from the superior edge of the pancreas and a Penrose drain was used to elevate the pancreas from the mesenteric vessels. Then, the pancreas was transected in a slow stepwise compression technique with a linear stapler using staple line reinforcement just to the left of the gastroduodenal artery. Finally, the splenic artery and splenic vein were dissected out and individually ligated using a linear stapler. The pancreas and spleen were then dissected away from the retroperitoneum and placed into a retrieval bag and removed.

For CP, eight operations were performed by laparoscopic technique and two operations were performed open. Similar to SP, once the lesion in the neck of the pancreas was identified, a Penrose drain was placed, and the neck of the pancreas was elevated and a linear stapler with staple line reinforcement was used to transect the pancreas just to the left of the gastroduodenal artery. A partial pancreatectomy of the neck (and possible proximal body) to include the lesion was then performed. The specimen was then removed, and frozen section analysis was used to ensure complete margin negative removal and confirm the absence of aggressive neoplasm. Reconstruction was then performed by implanting the left sided remnant pancreas into the posterior gastric wall for seven patients, and by the creation of a Roux-en-Y jejunal limb with a two-layer duct 
Table 1 Demographic data of 20 patients undergoing CP and SP

\begin{tabular}{|c|c|c|c|}
\hline Variable & $\mathrm{CP}(\mathrm{n}=10)$ & $S P(n=10)$ & $P$ value \\
\hline Mean age years ${ }^{\star}$ & $54.1(24.9-74.4)$ & $57.8(38.3-74.7)$ & 0.5127 \\
\hline Body mass index* & $32.5(44.3-21.7)$ & $27.3(17.0-32.0)$ & 0.0686 \\
\hline \multicolumn{4}{|l|}{ Comorbidities } \\
\hline NAFLD & $2(20 \%)$ & 0 & 0.4737 \\
\hline Diabetes mellitus (DM) & $3(30 \%)$ & $1(10 \%)$ & 0.5820 \\
\hline Pre-DM* & $2(20 \%)$ & 0 & \\
\hline DM with oral med & 0 & $1(10 \%)$ & \\
\hline Cardiac disease & 0 & 0 & \\
\hline Pulmonary disease & $3(30 \%)$ & $1(10 \%)$ & 0.5820 \\
\hline Significant history of alcohol use & $3(30 \%)$ & $2(20 \%)$ & 1.00 \\
\hline Tobacco use & $3(30 \%)$ & $4(40 \%)$ & 1.00 \\
\hline \multicolumn{4}{|l|}{ ECOG score } \\
\hline 0 & $9(90 \%)$ & $9(90 \%)$ & 1.000 \\
\hline 1 & $1(10 \%)$ & 0 & 1.00 \\
\hline 2 & 0 & 1 (10\%) & 1.00 \\
\hline
\end{tabular}

CP, central pancreatectomy; SP, subtotal pancreatectomy; NAFLD, Non-alcoholic fatty liver disease; ${ }^{*}$, pre-DM refers to diet-controlled diabetes; ECOG, Eastern Cooperative Oncology Group; ASA, American Society of Anesthesia.

to mucosa anastomosis in three patients. Surgical drains were left in place for all patients.

\section{Results}

A total of 809 patients underwent pancreatectomy at Mayo Clinic, Florida in this time period and of these, 10 patients $(1.2 \%)$ underwent CP. Both CP and SP resulted in definitive treatment of the underlying pathology. Table 1 includes the demographics of the groups. Overall, they were well matched although the patients undergoing $\mathrm{CP}$ had a non-significantly higher BMI than those undergoing SP. There were no missing variable data entries.
Table 2 gives operative variables for the groups. There was no significant difference in estimated blood loss nor in mean length of stay between CP and SP. There is a significant difference between $\mathrm{CP}$ and $\mathrm{SP}$ in average operative time $(\mathrm{CP}=312$ vs. $\mathrm{SP}=206, \mathrm{P}=0.0170)$.

Indications for resection in all 20 consecutive pancreatectomies included pancreatic neuroendocrine tumors, pancreatic cystic diseases, and other benign subtypes (Table 3). Pancreatic cystic diseases included includes intraductal papillary mucinous neoplasm (IPMN), mucinous cystic neoplasm (MCN), and solid pseudopapillary neoplasm (SPN). Benign subtypes include benign strictures and acute pancreatitis. $60 \%$ of $\mathrm{CP}$ patients 
Table 2 Operative data of 20 patients undergoing CP and SP

\begin{tabular}{lccc}
\hline Variable & CP $(\mathrm{n}=10)$ & $\mathrm{SP}(\mathrm{n}=10)$ & $\mathrm{P}$ value \\
\hline Operative time, min* & $312(217$ to 499$)$ & $206(116$ to 452$)$ & 0.0170 \\
Estimated blood loss, $\mathrm{mL}^{*}$ & $52(15$ to 100$)$ & 138 (0 to 600$)$ & 0.1409 \\
Length of stay, days * & $8(4$ to 29$)$ & $4.4(2$ to 9$)$ & 0.1397 \\
\hline
\end{tabular}

$\mathrm{CP}$, central pancreatectomy; SP, subtotal pancreatectomy; *, values are means.

Table 3 Pathological indications for resection of 20 patients undergoing $\mathrm{CP}$ and SP

\begin{tabular}{lccc}
\hline Variable & $\mathrm{CP}(\mathrm{n}=10)$ & $\mathrm{SP}(\mathrm{n}=10)$ & $\mathrm{P}$ value \\
\hline Pancreatic neuroendocrine tumor & 6 & 3 & 0.3698 \\
Pancreatic cystic disease & 3 & 6 & 0.3698 \\
Other benign subtype & 1 & 1 & 1.00 \\
\hline
\end{tabular}

$\mathrm{CP}$, central pancreatectomy; SP, subtotal pancreatectomy.

Table 4 Postoperative (90 days) complications of 20 patients undergoing CP and SP

\begin{tabular}{|c|c|c|c|}
\hline Variable & $\mathrm{CP}(\mathrm{n}=10)$ & $\operatorname{SP}(n=10)$ & $P$ value \\
\hline Pancreatic fistula & $8(80 \%)$ & 0 & 0.0007 \\
\hline$A$ & $4(40 \%)$ & 0 & \\
\hline $\mathrm{B}$ & $4(40 \%)$ & 0 & \\
\hline Post-pancreatectomy hemorrhage & $4(40 \%)$ & $1(10 \%)$ & 0.3034 \\
\hline$A$ & 0 & $1(10 \%)$ & \\
\hline $\mathrm{B}$ & $4(40 \%)$ & 0 & \\
\hline C & 0 & 0 & \\
\hline $\mathrm{B}$ & $1(10 \%)$ & 0 & 0 \\
\hline C & 0 & 0 & 0 \\
\hline Overall Morbidity (Clavien grade I-V) & $9(90 \%)$ & $4(40 \%)$ & 0.0573 \\
\hline Morbidity minor (Clavien grade I \& II) & $4(40 \%)$ & $4(40 \%)$ & 1.00 \\
\hline Morbidity major (Clavien grade III \& IV) & $5(50 \%)$ & 0 & 0.0325 \\
\hline
\end{tabular}

$\mathrm{CP}$, central pancreatectomy; SP, subtotal pancreatectomy.

had neuroendocrine tumors whereas $60 \%$ of SP patients had pancreatic cystic disease.

Postoperative (90 days) complications are found in Table 4. CP had a significantly higher POPF rate $(80 \%$ vs. $0 \%)$ and major morbidity rate $(50 \%$ vs. $0 \%)$ than patients undergoing SP. There were no mortalities in either group and all patients were still alive at the time of the study.

As seen in Table 5, there were no significant difference between incidence of DM among SP and CP patients. However, SP was noted to induce a higher proportion of 
Table 5 Pre-operative and long-term (greater than 6 months post-op) endocrine and exocrine insufficiency outcomes of 20 patients undergoing $\mathrm{CP}$ and SP

\begin{tabular}{|c|c|c|c|c|c|c|}
\hline Variable & \multicolumn{3}{|c|}{ Pre-op } & \multicolumn{3}{|c|}{ Post-op } \\
\hline \multicolumn{7}{|l|}{ Endocrine insufficiency } \\
\hline DM & $3(30 \%)$ & $1(10 \%)$ & 0.5280 & $4(40 \%)$ & 5 (50\%) & 1.00 \\
\hline Pre-DM & $2(20 \%)$ & 0 & & 0 & 0 & \\
\hline Insulin dependent DM & $1(10 \%)$ & 0 & & $2(20 \%)$ & 5 (50\%) & \\
\hline \multicolumn{7}{|l|}{ Exocrine insufficiency } \\
\hline Steatorrhea & 0 & 0 & 1.00 & $1(10 \%)$ & 0 & 1.00 \\
\hline Use of PERT & 0 & $1(10 \%)$ & 1.00 & 0 & $2(20 \%)$ & 0.4737 \\
\hline
\end{tabular}

CP, central pancreatectomy; SP, subtotal pancreatectomy; DM, diabetes mellitus; PERT, pancreas enzyme replacement therapy.

Table 6 Comparison of average follow-up time post-op and current BMI of 20 patients undergoing CP and SP

\begin{tabular}{lccc}
\hline Variable & $\mathrm{CP}(\mathrm{n}=10)$ & $\mathrm{SP}(\mathrm{n}=10)$ & $\mathrm{P}$ value \\
\hline Follow-up, months* & $95.2(3.5-259.4)$ & $64.2(2.9-154.1)$ & 0.3032 \\
Current BMI $^{*}$ & $33.3(21.7-45.0)$ & $26.9(17.3-31.7)$ & 0.0418 \\
\hline
\end{tabular}

BMI, Body Mass Index; CP, central pancreatectomy; SP, subtotal pancreatectomy; ${ }^{*}$, values are mean.

conversion to DM after surgery than CP (4 vs. 2 patients, $\mathrm{P}=0.6285)$. One patient was a diet-controlled diabetic prior to CP but became a non-diabetic post operation with follow-up at 7 months and confirmed with a HbA1c level of 5.7. Additionally, there were no significant difference in incidence of steatorrhea nor use of PERT enzymes between SP and CP patients postoperatively. One patient who underwent SP was on PERT prior to surgery.

In long term follow up, BMI was noted to slightly increase for patients undergoing $\mathrm{CP}$ while slightly decrease for those undergoing SP and become significantly different (26.9 vs. 33.3, $\mathrm{P}=0.0418$ ) in long term follow up (Table 6).

\section{Discussion}

The aim of this study is to assess the short-term complications and long-term post-operative endocrine and exocrine insufficiencies of CP and SP. Our data shows that there is a significantly higher rate of POPF $(80 \%$ vs. $0 \%, \mathrm{P}=0.007)$ and higher rate of major morbidity $(50 \%$ vs. $0 \%, \mathrm{P}=0.0325)$ in $\mathrm{CP}$ patients. The data does not, however, show any apparent advantage with either operation concerning long-term endocrine and exocrine insufficiencies. There is no significant difference between diabetic conversions ( $\mathrm{CP}=20 \%$, $\mathrm{SP}=40 \%$, $\mathrm{P}=0.6285$ ) nor in acquired exocrine insufficiency.

A meta-analysis evaluating 1305 patients undergoing CP revealed a higher morbidity and POPF but with lower rates of endocrine and exocrine that those undergoing DP. The study did not evaluate SP outcomes, however (9). There is significant variability in determining the safety and effectiveness of CP and SP in previous literature, specifically in the rates of POPF. Variation in POPF after CP ranges widely from $0 \%$ to $63 \%(2,9-12)$. Studies have noted occurrence of POPF in SP patients ranging from $14 \%$ to $20.6 \%(13,14)$. Studies show considerable differences in diabetic conversation rates ranging from $4 \%$ to $14 \%$ in CP and $14 \%$ to $35 \%$ in SP $(2,9,10,14)$. Rates of new-onset and worsening DM have been shown to have significant differences in $\mathrm{CP}$ and SP $(\mathrm{CP}=14 \%, \mathrm{SP}=46 \%$, $\mathrm{P}=0.003$ ) (10). Our institution has a relatively low morbidity and incidence of POPF after left pancreatectomy compared to other centers (8), however, the POPF of CP was noted to be high in this study and is being further reviewed with expected technical improvements to follow.

The range of differences between rates of CP and SP diabetic conversions may be due to a multitude of factors 
including remnant parenchyma volume and pancreatic adaptiveness. Studies estimate that no more than $20 \%$ to $25 \%$ of pancreas parenchyma is needed to establish sufficient glucose homeostasis for proper pancreatic function (15). New onset pancreatogenic diabetes has been shown to occur when more than $90 \%$ of the pancreas is resected (16). Pancreatic beta cells and islet cells are heavily concentrated in the body and tail of the pancreas which are resected in SP (14). This may explain the trends towards the observation of increased endocrine insufficiency in SP and distal pancreatectomy as compared to CP. However, it is important to note that islet cells gain functional adaptation following resection of the pancreas (15). Studies demonstrate adaptive increases in beta cell mass in response to diabetic adults (17). A study focused on glucose metabolism after SP for adenocarcinoma showed improved postoperative glucose tolerance and glucose metabolic capacity in all patients (18).

Postoperative BMI proved to be significantly higher in $\mathrm{CP}$ patients than in $\mathrm{SP}(\mathrm{CP}=33.27, \mathrm{SP}=26.89, \mathrm{P}=0.0418)$. DiNorcia et al. showed no difference in post-operative exocrine function as a function of gastrointestinal function, weight loss, and use of PERT between CP and distal pancreatectomy (10). Body weight has been observed to be significantly lower post-op in distal pancreatectomy patients compared to $\mathrm{CP}(2)$. The severing of nerve plexuses during pancreatectomy is not specific to CP nor SP and is ultimately consigned to the anatomy of the patient and the experience of the surgeon. This resection near the central neural ganglia of the celiac and superior mesenteric artery makes it important to compare $\mathrm{CP}$ to a SP with resection at the neck, rather than to distal pancreatectomy with no dissection near the central region. Exocrine insufficiency can be treated by the use of PERT which has been observed to reduce the extent of steatorrhea and abdominal pain post-pancreatectomy (19).

Consideration for CP should be to those patients who are obese and young with and have a higher risk of developing DM in the future. CP is best served by younger, fitter patients due to its significantly higher morbidity and incidence of complication. Older patients who may not be able to tolerate the increased short-term morbidity are best served by SP. A study showed no differences in mortality post SP between elderly patients $(>70)$ and young patients, citing SP as a safe procedure unhindered by age (20). BMI can be taken into consideration as a predictive factor of predicting new-onset DM. Hirata et al. found $\mathrm{BMI} \geq 25$ to be an independent risk factor of new- onset DM in those undergoing distal pancreatectomy or pancreaticoduodenectomy (21). Hwang et al. examined subtotal pancreatectomies and found an association between BMI >23 and impaired glucose tolerance postoperatively (14).

Due to the nature of this retrospective single institution study, several limitations were observed. First, patients were not able to be randomly assigned. While patient matching was used to counteract the absence of randomization, this proved to be difficult due to the relative rarity of $\mathrm{CP}$ and SP also affecting generalizability. Distal pancreatectomy was much more common, but we felt that this was not an adequate comparative procedure to CP. It is also difficult to properly assess long-term endocrine and exocrine insufficiency without follow-up protocol. Our study would have benefitted from a standard follow-up protocol, allowing for continuous review of critical data such as HbAlc levels, body weight, PERT use, and incidence of steatorrhea to better monitor changes in long-term endocrine/exocrine insufficiency. The small sample size of this study is a variable which should be taken into consideration when assessing long-term endocrine differences in SP and CP. Though the difference in diabetic conversions between SP and CP in our study is not significant, the trend towards increased rate of the development of diabetes must be taken into consideration. Overall, the small sample size of this study may have led to a type 2 statistical error. Further multi-institutional studies with much larger patient numbers must be undertaken in order to fully elucidate the short- and long-term comparison of CP versus SP.

$\mathrm{CP}$ and SP are considerable options to remove lowgrade localized tumors of the neck of the pancreas. CP is associated with higher morbidity than SP but may have slight long-term advantages. $\mathrm{CP}$ can be considered for younger obese patients prone to developing $\mathrm{DM}$ in the future. SP should be considered for aggressive tumors in which risk of malignancy and lymph node disease may compromise the effectiveness of the limited resection in CP. Older patients who may not be able to sustain the onset of short-term complication such as POPF and hemorrhage may be better served by SP.

\section{Acknowledgments}

Funding: None.

\section{Footnote}

Reporting Checklist: The authors have completed the 
STROBE reporting checklist. Available at https://jovs. amegroups.com/article/view/10.21037/jovs-21-16/rc

Data Sharing Statement: Available at https://jovs.amegroups. com/article/view/10.21037/jovs-21-16/dss

Conflicts of Interest: All authors have completed the ICMJE uniform disclosure form (available at https://jovs. amegroups.com/article/view/10.21037/jovs-21-16/coif). JAS serves as an unpaid editorial board member of fournal of Visualized Surgery from April 2020 to March 2022. The other authors have no conflicts of interest to declare.

Ethical Statement: The authors are accountable for all aspects of the work in ensuring that questions related to the accuracy or integrity of any part of the work are appropriately investigated and resolved. The study was conducted in accordance with the Declaration of Helsinki (as revised in 2013). The study was approved by institutional review board (IRB\#21-005622) and individual consent for this retrospective analysis was waived.

Open Access Statement: This is an Open Access article distributed in accordance with the Creative Commons Attribution-NonCommercial-NoDerivs 4.0 International License (CC BY-NC-ND 4.0), which permits the noncommercial replication and distribution of the article with the strict proviso that no changes or edits are made and the original work is properly cited (including links to both the formal publication through the relevant DOI and the license). See: https://creativecommons.org/licenses/by-nc-nd/4.0/.

\section{References}

1. Christein JD, Smoot RL, Farnell MB. Central pancreatectomy: a technique for the resection of pancreatic neck lesions. Arch Surg 2006;141:293-9.

2. Hirono S, Tani M, Kawai M, et al. A central pancreatectomy for benign or low-grade malignant neoplasms. J Gastrointest Surg 2009;13:1659-65.

3. Bassi C, Marchegiani G, Dervenis C, et al. The 2016 update of the International Study Group (ISGPS) definition and grading of postoperative pancreatic fistula: 11 Years After. Surgery 2017;161:584-91.

4. Wente MN, Veit JA, Bassi C, et al. Postpancreatectomy hemorrhage (PPH): an International Study Group of Pancreatic Surgery (ISGPS) definition. Surgery 2007;142:20-5.
5. Wente MN, Bassi C, Dervenis C, et al. Delayed gastric emptying (DGE) after pancreatic surgery: a suggested definition by the International Study Group of Pancreatic Surgery (ISGPS). Surgery 2007;142:761-8.

6. Asbun HJ, Stauffer JA. Laparoscopic vs open pancreaticoduodenectomy: overall outcomes and severity of complications using the Accordion Severity Grading System. J Am Coll Surg 2012;215:810-9.

7. Tsamalaidze L, Stauffer JA. Pancreaticoduodenectomy: minimizing the learning curve. J Vis Surg 2018;4:64.

8. Asbun HJ, Van Hilst J, Tsamalaidze L, et al. Technique and audited outcomes of laparoscopic distal pancreatectomy combining the clockwise approach, progressive stepwise compression technique, and staple line reinforcement. Surg Endosc 2020;34:231-9.

9. Xiao W, Zhu J, Peng L, et al. The role of central pancreatectomy in pancreatic surgery: a systematic review and meta-analysis. HPB (Oxford) 2018;20:896-904.

10. DiNorcia J, Ahmed L, Lee MK, et al. Better preservation of endocrine function after central versus distal pancreatectomy for mid-gland lesions. Surgery 2010;148:1247-54; discussion 1254-6.

11. Adham M, Giunippero A, Hervieu V, et al. Central pancreatectomy: single-center experience of 50 cases. Arch Surg 2008;143:175-80; discussion 180-1.

12. Zhou YM, Zhang XF, Wu LP, et al. Pancreatic fistula after central pancreatectomy: case series and review of the literature. Hepatobiliary Pancreat Dis Int 2014;13:203-8.

13. Ihse I, Anderson H, Andrén-Sandberg. Total pancreatectomy for cancer of the pancreas: is it appropriate? World J Surg 1996;20:288-93; discussion 294.

14. Hwang HK, Park J, Choi SH, et al. Predicting newonset diabetes after minimally invasive subtotal distal pancreatectomy in benign and borderline malignant lesions of the pancreas. Medicine (Baltimore) 2017;96:e9404.

15. King J, Kazanjian K, Matsumoto J, et al. Distal pancreatectomy: incidence of postoperative diabetes. J Gastrointest Surg 2008;12:1548-53.

16. Sakata N, Egawa S, Rikiyama T, et al. Computed tomography reflected endocrine function of the pancreas. J Gastrointest Surg 2011;15:525-32.

17. Butler PC, Meier JJ, Butler AE, et al. The replication of beta cells in normal physiology, in disease and for therapy. Nat Clin Pract Endocrinol Metab 2007;3:758-68.

18. Permert J, Ihse I, Jorfeldt L, et al. Improved glucose metabolism after subtotal pancreatectomy for pancreatic cancer. Br J Surg 1993;80:1047-50.

19. Czakó L, Takács T, Hegyi P, et al. Quality of life 
assessment after pancreatic enzyme replacement therapy in chronic pancreatitis. Can J Gastroenterol 2003;17:597-603.

20. al-Sharaf K, Andrén-Sandberg A, Ihse I. Subtotal pancreatectomy for cancer can be safe in the elderly. Eur J
Surg 1999; 165:230-5.

21. Hirata K, Nakata B, Amano R, et al. Predictive factors for change of diabetes mellitus status after pancreatectomy in preoperative diabetic and nondiabetic patients. J Gastrointest Surg 2014;18:1597-603.

doi: 10.21037/jovs-21-16

Cite this article as: Hanchate K, Tice M, Yeager TE, Stauffer JA. Central versus subtotal pancreatectomy: an assessment of short and long-term outcomes. J Vis Surg 2021;7:25. 